\title{
A Rare Cause of Ascites: Eosinophilic Gastroenteritis
}

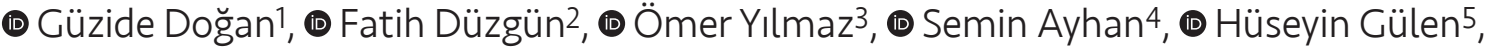 \\ (1) Erhun Kasırga1 \\ ${ }^{1}$ Celal Bayar University Faculty of Medicine, Department of Pediatric Gastroenterology Hepatology and Nutrition, Manisa, Turkey \\ ${ }^{2}$ Celal Bayar University Faculty of Medicine, Department of Radiology, Manisa, Turkey \\ ${ }^{3}$ Celal Bayar University Faculty of Medicine, Department of Pediatric Surgery, Manisa, Turkey \\ ${ }^{4}$ Celal Bayar University Faculty of Medicine, Department of Pathology, Manisa, Turkey \\ ${ }^{5}$ Celal Bayar University Faculty of Medicine, Department of Pediatric Hematology, Manisa, Turkey
}

\section{ABSTRACT}

Eosinophilic gastroenteropathies are rare disorders. An 18-year-old girl presented to the hospital with abdominal pain and distension. Abdominal tenderness and grade 2 ascites, serious peripheral eosinophilia and high immunoglobulin E level were found. Laparoscopic antrum biopsy showed subserosal eosinophilic infiltration. Eosinophilic gastroenteropathy was suspected, diet elimination was given, no steroid treatment was used.

Keywords: Eosinophilic gastroenteritis, eosinophilia, ascites, children

\section{Introduction}

Eosinophilic gastroenteropathies (EoGs) are rare and poorly defined diseases of the gastrointestinal tract (GIT). These disorders, which are usually associated with peripheral eosinophilia, involve recurrent eosinophilic infiltration of at least one organ within the GIT $(1,2)$. Symptoms are similar to those of many systemic diseases. Gastroenterologists and general internal medicine specialists, therefore, should seek more awareness to ensure a proper diagnosis is made.

\section{Case Presentation}

An 18-year-old female presented to the hospital having had generalized abdominal pain and swelling for a week with no identified relation to food. Nausea and vomiting were reported during the previous three days.
She did not demonstrate any fever, weight loss, night sweats, chest pain, or joint swelling and was not taking any prescribed medication or herbal compounds. Upon physical examination, increased intestinal sounds accompanied by slight abdominal swelling, abdominal tenderness without rebound and grade 2 ascites were noted. Her white blood cell count was found to be $15,200 / \mu \mathrm{L}$ with $58.4 \%$ being $(8,900 / \mu \mathrm{L})$ eosinophils. Biochemistry, thyroid function, sediment and urine analysis findings were all normal. Additionally, C-reactive protein, anti-endomysial antibodies, tissue transglutaminase antibody, anti-nuclear antibody, anti-ds DNA, anti-mitochondria antibody tests and urine culture results were all negative, while her immunoglobulin E (IgE) level was $1,321 \mathrm{IU} / \mathrm{mL}$. Stool parasites tests and toxocara, echinococcus and faecal calprotectin tests were also negative. Flow cytometry of a peripheral blood sample

\section{Address for Correspondence}

Güzide Doğan MD, Celal Bayar University Faculty of Medicine, Department of Pediatric Gastroenterology Hepatology and Nutrition, Manisa, Turkey Phone: +905058219424 E-mail: guzidedogan@gmail.com ORCID: orcid.org/0000-0003-4291-7282 Received: 07.10.2018 Accepted: 08.01.2019

${ }^{(\odot)}$ Copyright 2020 by Ege University Faculty of Medicine, Department of Pediatrics and Ege Children's Foundation The Journal of Pediatric Research, published by Galenos Publishing House. 
did not reveal any myelolymphoproliferative findings. A contrasted abdominal computed tomography (CT) coronal section scan showed moderate ascites with thickened gastric antrum and small bowel walls (Figure 1). Abdominal paracentesis revealed that $95 \%$ of white blood cells were eosinophils (Figures $2 \mathrm{a}$ and $2 \mathrm{~b}$ ). Acid samples were as follows: $\mathrm{pH}=7.47$; lactate dehydrogenase $=401 \mathrm{mg} / \mathrm{dL}$; and albumin= $3.18 \mathrm{~g} / \mathrm{dL}$ (serum albumin= $4.0 \mathrm{~g} / \mathrm{dL}$ ). Skin prick test was negative. Esophagogastroduodenoscopy (EGD) demonstrated moderate pangastritis, bulbitis, and duodenitis. Histological appearance revealed 17 to 20 eosinophils per high-power field of the oesophagus and chronic non-atrophic gastritis (Figure 2c). Histological findings from the gastric mucosa did not reveal eosinophilic gastritis; thus, laparoscopy was performed. During the laparoscopic examination, a hyperaemic oedema area of 2 $\mathrm{cm}$ to $3 \mathrm{~cm}$ was detected on the front of the gastric antrum (Figure 3). In the biopsy evaluation of the stomach antrum, eosinophilic infiltration of the prominent muscularis propria and subserosa and associated mild acute inflammation reaction were detected (Figure $2 \mathrm{~d}$ ).

As a consequence, a diagnosis of eosinophilic gastroenteritis was made. She was treated via the elimination of milk and milk products from the diet and proton pump inhibitor administration. Her abdominal pain, acid and examination findings were completely resolved

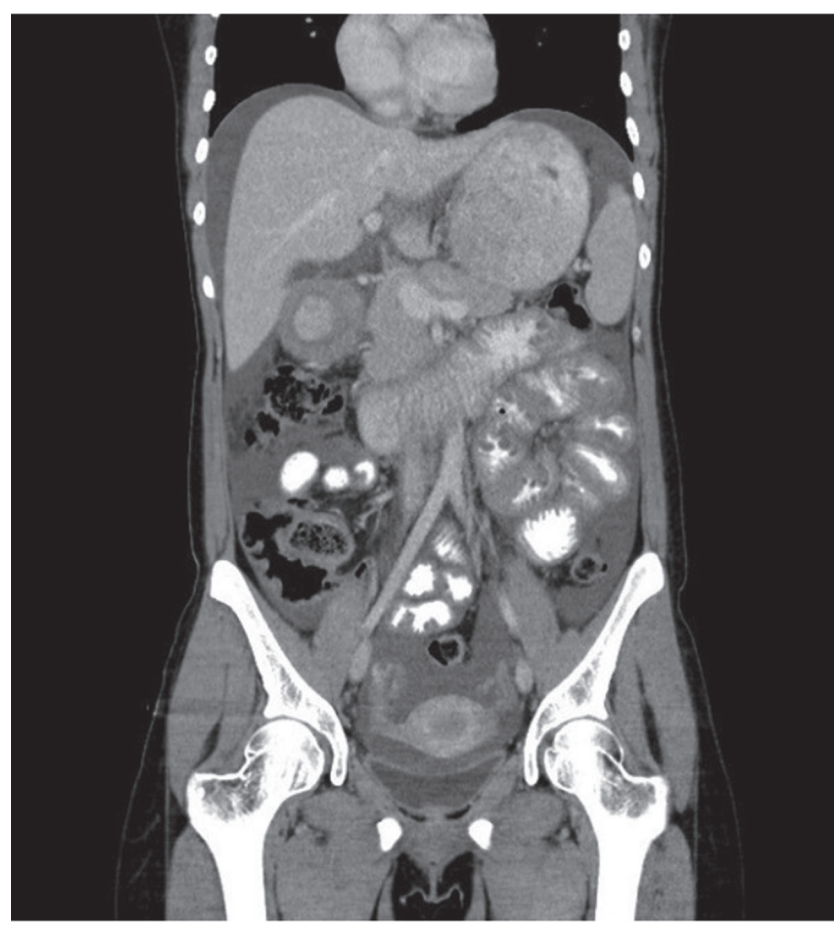

Figure 1. Abdominal computed tomography showed moderate ascites with thickening of the gastric antrum and small bowel walls after two weeks of milk and dairy products being eliminated. Her absolute eosinophil count at this point was $1,900 / \mu \mathrm{L}$ (25.7\%). Additionally, her IgE level was decreased to 202 $\mathrm{IU} / \mathrm{mL}$ and abdominal ultrasonography showed complete resolution of ascites. By the second month of treatment, she was asymptomatic while continuing the elimination diet. Her peripheral blood count revealed an absolute eosinophil count of $300 / \mu \mathrm{L}(8.3 \%)$.

\section{Discussion}

EoGs are an infrequently observed disease characterized by recurrent eosinophilic infiltration of various sections of the GIT. Eosinophilic tissue infiltration may affect any of the three layers (i.e., mucosal, muscular, subserosal) in the digestive tract wall, with symptoms typically varying according to the affected layer (3). Of the three types, the mucosal form is the most common, while the serosal form is the least common (1). Often, symptoms of EoGs include colonic abdominal pain, bloating, diarrhoea, weight loss, and vomiting $(4,5)$. Other features of severe EoGs are gastrointestinal bleeding, iron deficiency anaemia, hypoalbuminemia, protein-losing enteropathy, and growth disturbance (5). Patient symptoms in the case of the mucosal form of EoGs are usually abdominal pain, nausea, vomiting, and diarrhoea. The muscular form in contrast demonstrates a thickened intestine that match the symptoms of intestinal obstruction (6). Serosal infiltration accompanied by eosinophils and associated abdominal distension have rarely been reported with eosinophilic ascites and bowel perforation (4,7-9).

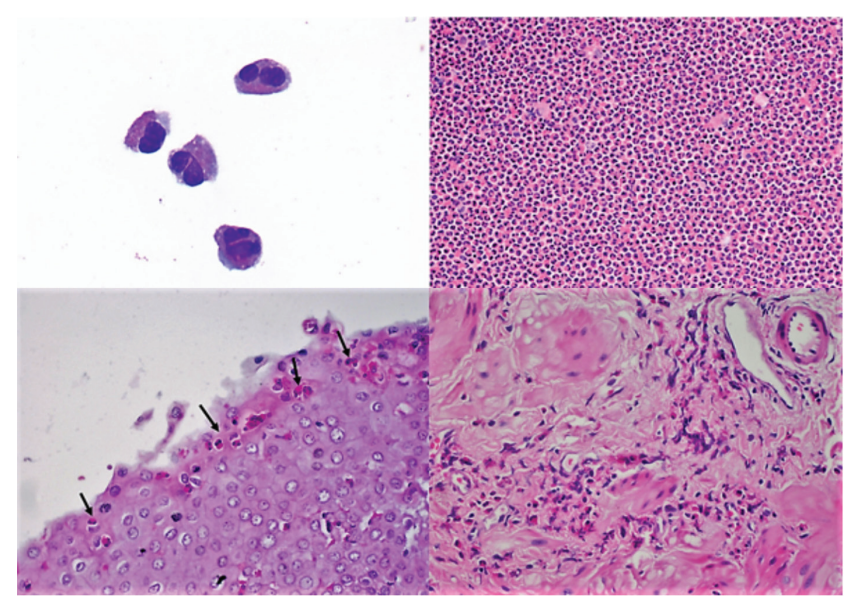

Figure 2. Figure 2a: High-power oil immersion view of the peripheral blood film shows eosinophils (Wright-Giemsastain; x1000). Figure 2b: Diagnostic paracentesis demonstrates ascitic fluid rich in eosinophils $(H \& E, x 40)$. Figure 2c: Eosinophiles scattered towards the surface of the mucosa in the endoscopic biopsyspecimen of esophageal mucosa ( $H \& E$, $\mathrm{x} 40$ ). Figure $2 \mathrm{~d}$ : Prominent eosinophilic infiltration in the intersitisiel connective tissue in the laparoscopic biopsy 
Peripheral eosinophilia is a very common finding in EoG (9). Eosinophilia was determined to be $58.4 \%$ in our case due to serosal involvement. When searching for other eosinophilia causes, the results of the relevant tests were negative. The patient was not taking any prescribed medications such as aspirin, penicillin, cephalosporins, cotrimoxazole, or carbamazepine. The patient refused colonoscopy, which would have been the next step in the clinical workup of eosinophilic gastroenteritis. Clinical, laboratory, and histological findings of our patient were not compatible with Crohn's disease. Ascites does not appear in most patients at the time of exacerbation of Crohn's disease (10).

An EoGs diagnosis is based on high clinical suspicion with histopathological findings. If the diagnosis is uncertain, CT imaging can help to determine the location of the thickened GIT areas. The presentation of ascites that develops in serosal involvement is exudative and eosinophil-rich, as detected in this patient (9). EGD examination can detect hyperaemia, ulceration, stricture, nodular appearance and ulcer in EoGs. Pangastritis, bulbitis and duodenitis were seen in this case. A full-thickness biopsy is taken laparoscopically for subserosal involvement and is sometimes necessary for diagnosis $(1,3)$. In this case, a seromuscular biopsy was obtained and the presence of a dense eosinophil infiltration in the biopsy specimen revealed a diagnosis of EoG (Figure 3).

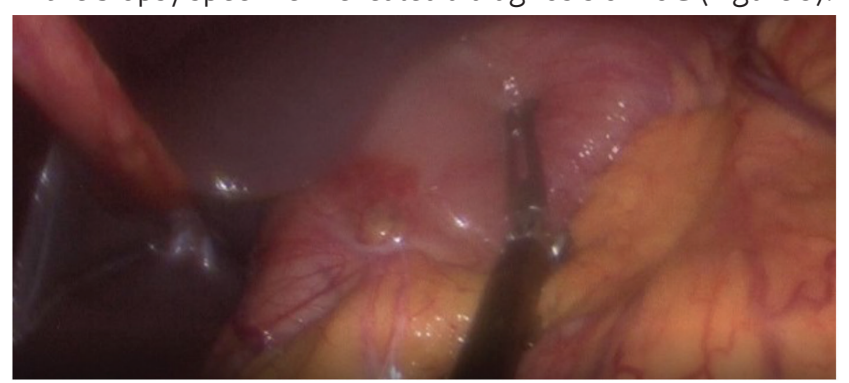

Figure 3. Laporoscopic evaluation of the patient revealed serosal inflammation involving the portion of antrum

\section{Conclusion}

The first-line treatment for EoG is the removal of the responsible food from the diet (1). However, nutritional elimination treatment is only valid in some patients, as there are difficulties inherent in identifying the responsible food (4). We treated the present case with food elimination only. Subsequently, ascites and eosinophilia reduction occurred. This is a different outcome from other studies that required steroid therapy $(7,9)$. Corticosteroids are an important treatment option if dietary therapy is unsuccessful in promoting remission, although they should be used with caution due to associated side effects and it should be kept in mind that relapses may occur at a later time after medication discontinuation (1). Therefore, nutritional elimination should be tried prior to the introduction of steroid therapy in clinically appropriate patients. Since an outcome similar to our case who responded to the elimination of nutrients alone had not been previously reported, we would like to present our report as documentation to contribute to the literature.

\section{Ethics}

Informed Consent: Informed consent was obtained.

Peer-review: Externally and internally peer-reviewed.

\section{Authorship Contributions}

Medical Practices: G.D., Ö.Y., E.K., Concept: G.D., H.G., Design: G.D., F.D., Data Collection or Processing: G.D., F.D., S.A., Analysis or Interpretation: G.D., S.A., H.G., Literature Search: G.D., E.K., Writing: G.D., F.D.

Conflict of Interest: No conflict of interest was declared by the authors.

Financial Disclosure: The authors declared that this study received no financial support.

\section{References}

1. Markowitz E), Liacouras CA. Allergic and Eosinophilic Gastrointestinal Disease. In: Wyllie R, Hyams JS, Kay M, eds. Pediatric Gastrointestinal and Liver Disease, 5th Edition Elsevier 2016; 429-40.

2. Heine RG. Pathophysiology, diagnosis and treatment of food protein-induced gastrointestinal diseases. Curr Opin Allergy Clin Immunol 2004; 4:221-9.

3. Talley NJ, Shorter RG, Phillips SF, Zinsmeister AR. Eosinophilic gastroenteritis: a clinicopathological study of patients with disease of the mucosa, muscle layer, and subserosal tissues. Gut 1990; 31:54-8.

4. Rodríguez Jiménez B, Domínguez Ortega J, González García JM, Kindelan Recarte C. Eosinophilic gastroenteritis due to allergy to cow's milk. I Investig Allergol Clin Immunol 2011; 21:150-2.

5. Kelly KJ. Eosinophilic gastroenteritis. J Pediatr Gastroenterol Nutr 2000; 30:28-35.

6. Chen MJ, Chu CH, Lin SC, Shih SC, Wang TE. "Eosinophilic gastroenteritis: clinical experience with 15 patients," World Journal of Gastroenterology 2003; 9:2813-6.

7. Santos J, Junquera F, de Torres I, Molero X, Vilaseca J, Malagelada JR. Eosinophilic gastroenteritis presenting as ascites and splenomegaly. Eur / Gastroenterol Hepatol 1995; 7:675-8.

8. Wang CS, Hsueh S, Shih LY. Repeated bowel resections for eosinophilic gastroenteritis with obstruction and perforation. Case report. Acta Chir Scand 1990; 156:333-6.

9. Lerza P. A further case of eosinophilic gastroenteritis with ascites. Eur I Gastroenterol Hepatol 1996; 8:407.

10. Aparicio Tormo JR, Laveda R, Gomez Andres A. Crohn's disease and ascites. Gastroenterol Hepatol 2000; 23:384-8. 